\title{
Downregulation of YAP inhibits proliferation and induces apoptosis in Eca-109 cells
}

\author{
MU CUI and ZHEN LI \\ School of Nursing, Xi'an Medical University, Xi'an, Shaanxi 710021, P.R. China
}

Received January 23, 2017; Accepted July 11, 2017

DOI: $10.3892 /$ etm.2017.5492

\begin{abstract}
A previous study reported that Yes-associated protein (YAP) gene was overexpressed in esophageal squamous cell carcinoma (ESCC); however, the exact role of YAP in ESCC remains largely unclear. The present study aimed to investigate the effects of YAP inhibition on ESCC. In order to investigate the exact role of YAP in ESCC cells, a stable YAP low-expression ESCC cell line was established using YAP-small interfering RNA. MTT assay was performed to examine the cell proliferation ability, while flow cytometry were used to detect the cell apoptosis and cell cycle distribution. In addition, reverse transcription-quantitative polymerase chain reaction and western blot analysis were applied for mRNA and protein level detection, respectively. The results suggested that YAP gene inhibition significantly repressed the ECA-109 cell proliferation and induced cell apoptosis, whereas this inhibition had no significant effects on cell cycle. Furthermore, the expression levels of cell apoptosis-associated proteins were determined in the current study, and the data demonstrated that the B-cell lymphoma 2 (Bcl-2)/Bcl-2-associated $\mathrm{X}$ protein ratio and phosphorylated extracellular signal-regulated kinase expression were significantly reduced, while the p53 and caspase 3 levels were notably increased in YAP gene-inhibited ECA-109 cells. In conclusion, the current study revealed that YAP gene inhibition suppresses the proliferation and induces apoptosis in ECA-109 cells, indicating that the YAP gene serves as an oncogene in ESCC.
\end{abstract}

\section{Introduction}

Esophageal cancer is one of the eight most common human tumors worldwide, which commonly occurs in the esophageal mucosal epithelium or gland, and its mortality rate ranked sixth worldwide (1,2). Approximately 482,300 patients were

Correspondence to: Dr Mu Cui, School of Nursing, Xi'an Medical University, 1 Xinwang Road, Xi'an, Shaanxi 710021, P.R. China E-mail: cuimu2016@sina.com

Key words: esophageal squamous cell carcinoma, Hippo/Yes-associated protein signaling pathway, oncogene, proliferation, apoptosis newly diagnosed and 406,800 patients succumbed to esophageal cancer worldwide in 2008 (3). This type of cancer has a high incidence in developing countries, mainly in East Asia, East Africa and South Africa. There are two main histological types of esophageal cancer, including the esophageal adenocarcinoma and the esophageal squamous cell carcinoma (ESCC), which accounts for $>90 \%$ of cases in China (4). Esophageal cancer is one of the malignant diseases that severely endanger the life and health of individuals in China. According to the investigation of the cancer incidence in China, esophageal cancer is the fifth most common type of cancer and the fourth highest cause of cancer-associated mortality (5).

In recent years, although advances have been achieved in surgery, chemotherapy and other treatment methods of esophageal cancer, the worldwide overall survival rate is $<10 \%$ and the 5 -year survival rate is only $14 \%$ due to the high recurrence rate of esophageal cancer and poor prognosis $(6,7)$. Therefore, at present, the research on the pathogenesis and treatment of esophageal squamous cell carcinoma is receiving increasing attention.

Yes-associated protein (YAP), a $65 \mathrm{kD}$ molecule, is the nuclear effector of the Hippo signaling pathway which is a newly discovered cell signal transduction pathway that serves essential roles in regulating the volume and size of organs $(8,9)$. YAP participates in tumor progression via regulating cell growth, proliferation and apoptosis, and is regarded as an oncogene (10). Previous studies have demonstrated that YAP is overexpressed in a variety of tumor tissues, including breast, liver, prostate, colon and lung cancer (11-15). It has also been indicated that YAP overexpression is frequently observed in ESCC and it may serve as an oncogene in ESCC (16).

The exact role of YAP in ESCC remains largely unclear. Therefore, the present study aimed to investigate the effects of YAP inhibition on ESCC by small interfering RNA (siRNA) transfection in ECA-109 cells, and to explore the underlying molecular mechanism.

\section{Materials and methods}

Cell culture. Human ECA-109 cells were obtained from the American Type Culture Collection (cat.no. EY-X0635; Manassas, VA, USA). The ECA-109 cells were grown in RPMI-1640 medium (Gibco; Thermo Fisher Scientific, Inc., Waltham, MA, USA) containing 10\% FBS (Gibco; Thermo Fisher Scientific, Inc.) and $1 \%$ penicillin-streptomycin solution. Cells were incubated in a humidified atmosphere with $5 \% \mathrm{CO}_{2}$ at $37^{\circ} \mathrm{C}$. 
Cell transfection. At $24 \mathrm{~h}$ prior to transfection, ECA-109 cells $\left(5 \times 10^{4}\right.$ cells per well) were seeded into a 6 -well plate and cultured at $37^{\circ} \mathrm{C}$ in $5 \% \mathrm{CO}_{2}$ humidified air. When the cell confluence reached 60-70\%, YAP-siRNA or control-siRNA (Santa Cruz Biotechnology, Inc., Dallas, TX, USA) was transfected into the cells using Lipofectamine 2000 (Invitrogen; Thermo Fisher Scientific, Inc.) according to the manufacturer's protocol. The cells were initially transfected for $12 \mathrm{~h}$, followed by further incubation for $24 \mathrm{~h}$ at $37^{\circ} \mathrm{C}$ in $5 \% \mathrm{CO}_{2}$. Subsequently, the culture medium was refreshed. Cells transfected with YAP-siRNA were considered as the siRNA group (siRNA), cells transfected with control-siRNA were considered to be the negative control group (NC), and cells without any treatment were considered to be the blank control group (BL).

MTT assay. Cells were seeded in a 96-well culture plate $\left(\sim 200 \mu \mathrm{l}\right.$ at $1 \times 10^{4}$ cells $\left./ \mathrm{ml}\right)$, and an MTT assay was performed at $24 \mathrm{~h}$ after transfection to determine the cell proliferation ability of cells. Briefly, MTT solution $(20 \mathrm{mg} / \mathrm{ml}$; Amresco LLC, Solon, OH, USA) was added into the culture medium in each well and incubated for $4 \mathrm{~h}$ at $37^{\circ} \mathrm{C}$. Next, the optical density (OD) at $570 \mathrm{~nm}$ was detected by a microplate reader.

Apoptosis analysis assay. At $24 \mathrm{~h}$ after transfection, cells were washed three times with cold phosphate-buffered saline (PBS) solution, fixed with $70 \%$ ethanol at room temperature for $15 \mathrm{~min}$ and rinsed again with PBS. Subsequently, the cells were labeled with Annexin V-FITC and propidium iodide (PI) (cat. no. 6592; Cell Signaling Technology, Danvers, MA, USA) following the manufacturer's protocol, and incubated at room temperature for $30 \mathrm{~min}$. Flow cytometry (FCM; BD Biosciences, Franklin Lakes, NJ, USA) was then applied to analyze the cell apoptosis. Experiments were performed in triplicate.

Cell cycle assay. At $24 \mathrm{~h}$ after transfection, the transfected ECA-109 cells were collected using $0.25 \%$ trypsin, washed three times with PBS solution and then fixed with $70 \%$ ethanol at $4^{\circ} \mathrm{C}$ overnight. Following centrifugation $\left(1,000 \times \mathrm{g}, 4^{\circ} \mathrm{C}\right.$ for, $5 \mathrm{~min}$ ) and washing, the cells were dyed with RNAse $\mathrm{A}$ and PI and incubated at $4^{\circ} \mathrm{C}$ for $30 \mathrm{~min}$. The cell cycle distribution was then analyzed using a flow cytometer (FACSort; FACSCanto II; BD Biosciences, Franklin Lakes, NJ, USA).

Western blot analysis. The protein expression levels of YAP, B-cell lymphoma 2 (Bcl-2), Bcl-2-associated X protein (Bax), p53, caspase 3, extracellular signal-regulated kinase (Erk) and phosphorylated Erk (p-Erk) were determined by western blot analysis. Briefly, the total cell protein was collected from the transfected cells using a lysis buffer (Cell Signaling Technology). Next, a BCA assay (Thermo Fisher Scientific, Inc.) was applied to detect the protein concentrations of the cell lysates, and bovine albumin was used as standard. Protein samples were then separated by $10 \%$ SDS-PAGE and then blotted onto polyvinylidene difluoride membranes, followed by blocking with $5 \%$ fat-free powdered milk for $2 \mathrm{~h}$ at room temperature. Subsequently, the membranes were incubated overnight at $4^{\circ} \mathrm{C}$ with the primary antibodies against YAP (cat. no. 14074), Bcl-2 (cat. no. 4223), Bax (cat. no. 5023), p53 (cat. no. 2527), caspase 3 (cat.no. 9665), Erk (cat.no.4695) and p-Erk (cat. no. 8510; all 1:1,000; all from Cell Signaling Technology).

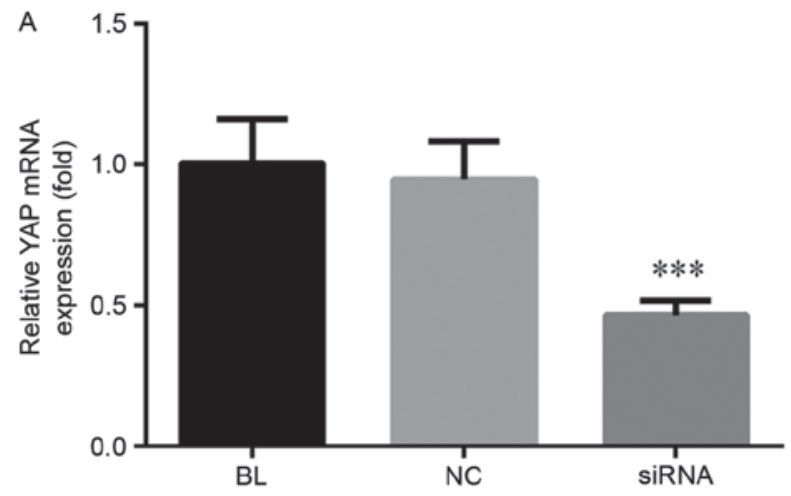

B

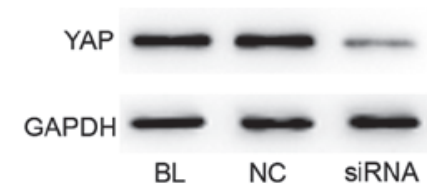

Figure 1. YAP expression in Eca-109 cells. At $24 \mathrm{~h}$ after transfection with YAP siRNA or control siRNA, (A) reverse transcription-quantitative polymerase chain reaction and $(B)$ western blot analysis were performed to detect the mRNA and protein expression levels of YAP, respectively. Data are presented as the mean \pm standard deviation. ${ }^{* * *} \mathrm{P}<0.001$ vs. BL group. YAP, Yes-associated protein; siRNA, small interfering RNA.

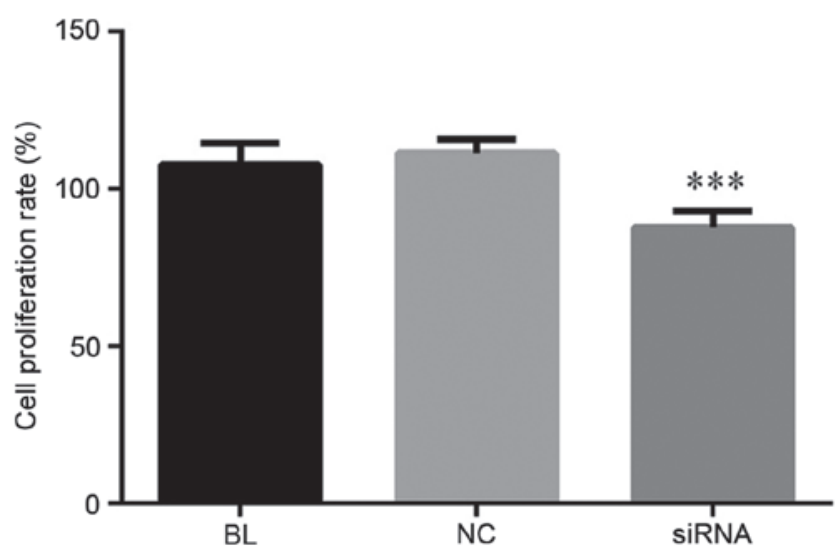

Figure 2. YAP inhibition suppresses Eca-109 cell proliferation. At $24 \mathrm{~h}$ after transfection, the cell proliferation ability of Eca-109 cells was determined by an MTT assay. Data are expressed as the mean \pm standard deviation. ${ }^{* * * *} \mathrm{P}<0.001$ vs. BL group. YAP, Yes-associated protein.

$\beta$-actin (cat. no. 4970; 1:5,000; Cell Signaling Technology) was used as an internal control. Following washing three times with Tris-buffered saline/Tween-20, the samples were then incubated with horseradish peroxidase-conjugated secondary antibodies for $2 \mathrm{~h}$ at room temperature. Finally, the membranes were developed with an enhanced chemiluminescence detection kit (cat. no. 6883; Cell Signaling Technology) according to the manufacturer's protocol.

Reverse transcription-quantitative polymerase chain reaction $(R T-q P C R)$. Total RNA was isolated from the transfected cells using TRIzol regent (Invitrogen; Thermo Fisher Scientific, Inc.) following the manufacturer's protocol. The RNA concentration was quantified by using a Nanodrop spectrophotometer (Thermo Fisher Scientific, Inc.) at $260 \mathrm{~nm}$. cDNAs were generated using the PrimeScript reverse transcription reagent 

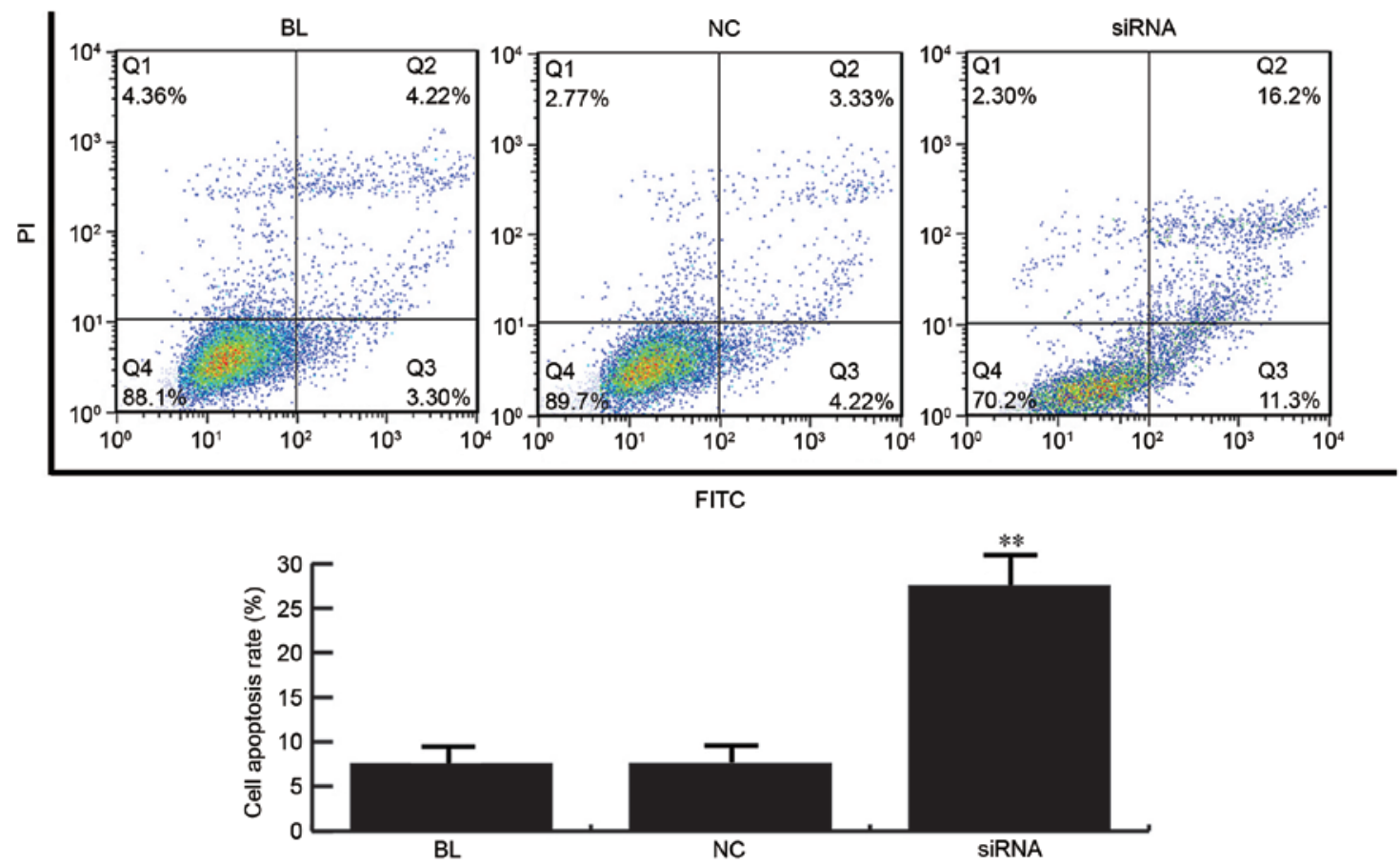

Figure 3. YAP inhibition induces Eca-109 cell apoptosis. At $24 \mathrm{~h}$ after transfection, the apoptosis of Eca-109 cells was determined by flow cytometry. Data are expressed as the mean \pm standard deviation. ${ }^{* *} \mathrm{P}<0.01$ vs. BL group. YAP, Yes-associated protein.

kit (Takara Biotechnology Co., Ltd., Dalian, China) according to the manufacturer's instructions. cDNAs were subsequently analyzed using qPCR with a TaqMan Universal PCR Master Mix kit and the ABI PRISM 7900 HT sequence-detection system (Thermo Fisher Scientific, Inc.). The amplification conditions were as follows: $95^{\circ} \mathrm{C}$ for $10 \mathrm{~min}$, followed by 40 cycles of $95^{\circ} \mathrm{C}$ for $10 \mathrm{sec}$ and $60^{\circ} \mathrm{C}$ for $60 \mathrm{sec}$. The primer sequences used in qPCR were as follows: YAP forward, 5'-ACGTTCATCTGGGACAGCAT-3' and reverse, 5'-GTT GGGAGATGGCAAAGACA-3'; GAPDH forward, 5'-GGC ATTGCTCTCAATGACAA-3' and reverse, 5'-TGTGAGGGA GATGCTCAGTG-3'. GAPDH was used as an internal control. The relative gene expressions were quantified using the $2^{-\Delta \Delta C q}$ method (17).

Statistical analysis. Experiments were performed for at least three times. SPSS 17.0 statistical software (SPSS, Inc., Chicago, IL, USA) was performed for all statistical analyses. The results are expressed as the mean \pm standard deviation, and were compared by Student's t-test or one-way analysis of variance followed by Tukey's test. Statistically significant differences were indicated by $\mathrm{P}<0.05$.

\section{Results}

YAP expression is inhibited by YAP siRNA transfection in ECA-109 cells. In order to investigate the exact role of YAP in ESCC cells, a stable YAP low-expression ESCC cell line was established using YAP siRNA transfection. RT-qPCR and western blot analysis were performed to detect the mRNA and protein YAP expression levels, respectively (Fig. 1). The results indicated that the mRNA and protein levels of YAP were significantly inhibited following YAP siRNA transfection in Eca-109 cells.
YAP inhibition suppresses cell proliferation in ECA-109 cells. At $24 \mathrm{~h}$ after transfection, the cell proliferation ability of Eca-109 cells was determined by MTT assay. As shown in Fig. 2, compared with the controls, the Eca-109 cell proliferation ability was notably decreased in cells transfected with YAP siRNA. These data indicated that YAP inhibition was able to suppress the proliferation in Eca-109 cells.

YAP inhibition induces cell apoptosis in ECA-109 cells. The effects of YAP on cell apoptosis were detected using FCM. YAP siRNA or control siRNA was transfected into Eca-109 cells, and the cell apoptosis was detected at $24 \mathrm{~h}$ after transfection. As observed in Fig. 3, the apoptosis rate of Eca-109 cells was significantly increased in the YAP siRNA-transfected group. Thus, these results indicated that YAP inhibition induced cell apoptosis in Eca-109 cells.

YAP inhibition exerts no effects on the cell cycle distribution in ECA-109 cells. At $24 \mathrm{~h}$ after transfection, the effects of YAP inhibition on the cell cycle were determined. As shown in Fig. 4, no significant difference was detected between the controls and the YAP siRNA group with regard to the percentage of cells at each cell cycle phase.

Effects of YAP inhibition on the expression of proteins associated with cell proliferation and apoptosis. In order to investigate the underlying molecular mechanisms of YAP-induced regulation of cell proliferation and apoptosis, western blot analysis was conducted to detect the expression levels of proteins associated with cell proliferation and apoptosis. The results suggested that, compared with the controls, the expression levels of Bcl-2 and p-Erk were evidently decreased in the YAP siRNA-transfected group, while the levels of Bax, caspase 3 and p53 were notably increased (Fig. 5). 


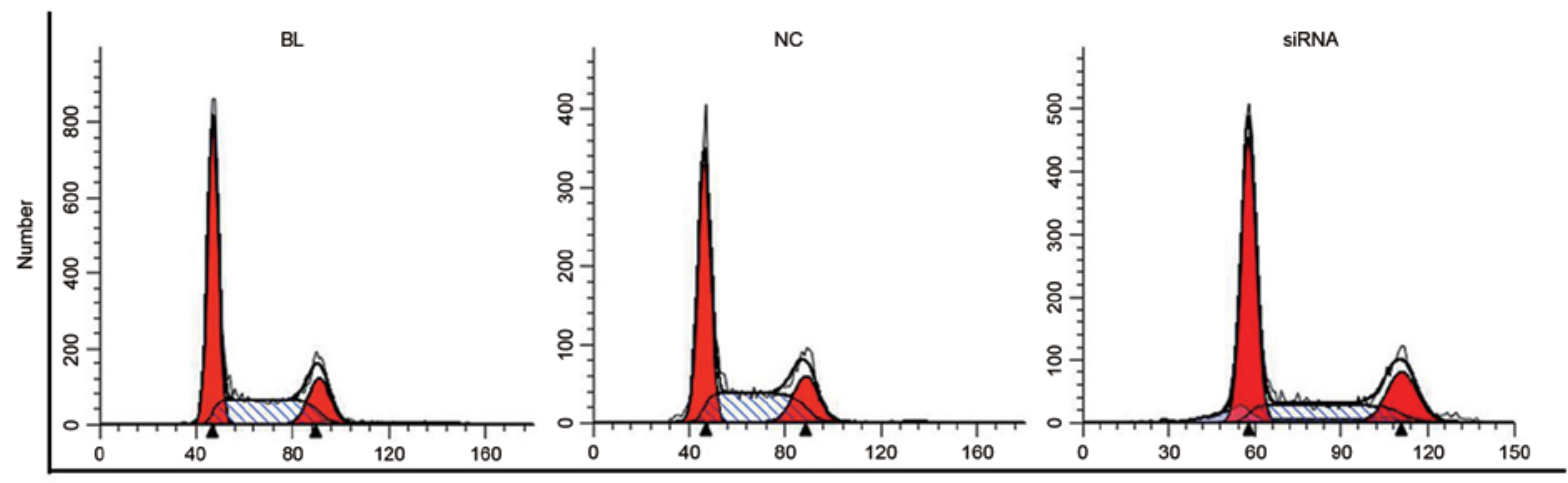

Channels (FL2-A)

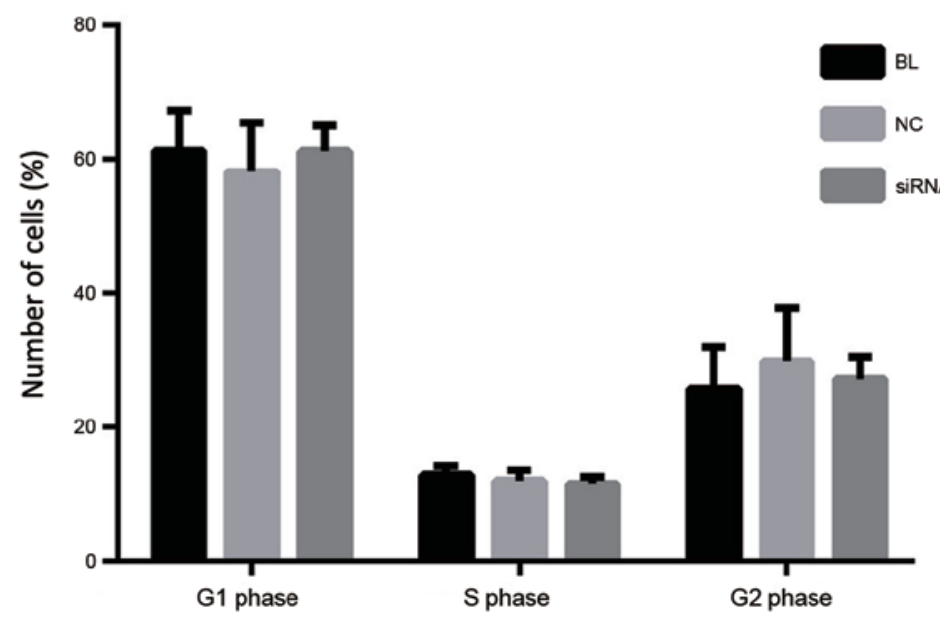

Figure 4. YAP inhibition causes no significant effect on Eca-109 cell cycle. At $24 \mathrm{~h}$ after transfection, the cell cycle distribution of Eca-109 cells was determined by flow cytometry. Data are presented as the mean \pm standard deviation. YAP, Yes-associated protein.

\section{Discussion}

The Hippo signaling pathway regulates the organ size in various species, and dysregulation of this pathway may induce the development of tumors (18). Since the disruption of any factor in this pathway can lead to tumorigenesis, YAP is recognized as an oncogene and the major downstream effector of the Hippo signaling pathway. Upregulation of the YAP gene and its nuclear localization have been observed in a variety of cancer types, including hepatocellular carcinoma (HCC), as well as lung, ovarian, prostate and colon cancer (19-22). It has been observed that YAP is an independent prognostic marker for the overall survival and disease-free survival of HCC patients (21). Furthermore, a previous study demonstrated that the overexpression and nuclear localization of YAP in ESCC tissues was associated with a poor prognosis (16).

RNA interference, a process of post-transcriptional regulation of gene expression, has been widely used in experiment studies $(23,24)$. In the present study, this process was used to investigate the effects of YAP inhibition on ESCC cells using YAP siRNA transfection. Subsequent to transfection with YAP siRNA, the mRNA and protein expression levels of YAP in Eca-109 cells were significantly decreased. MTT assay was also applied to determine the effect of YAP inhibition on Eca-109 cell proliferation, and the results suggested that YAP inhibition prevented the proliferation ability of the Eca-109 cells. The Eca-109 cell apoptosis and cell cycle distribution were also detected using FCM, which revealed

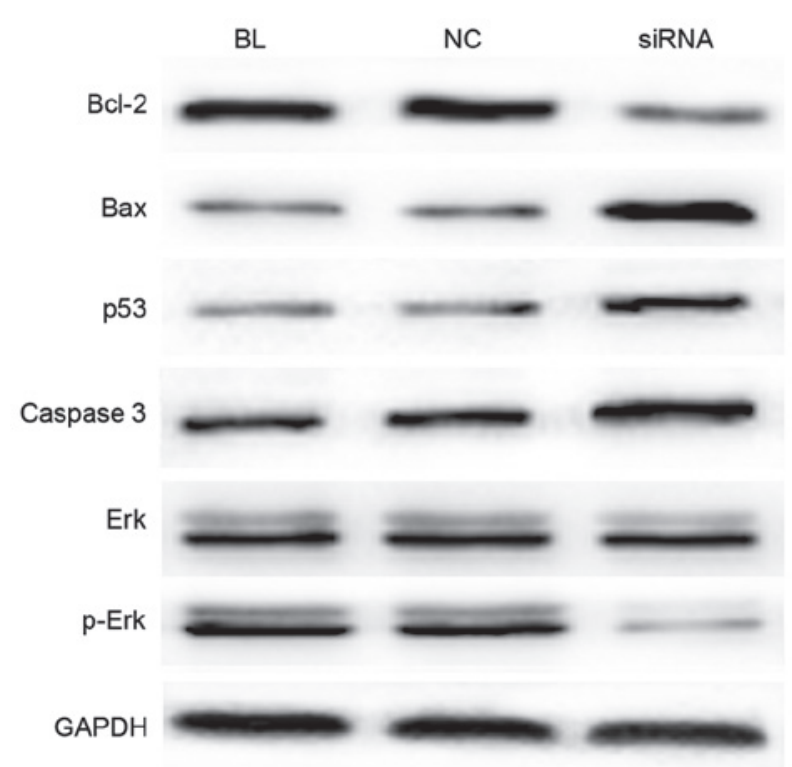

Figure 5. YAP inhibition alters the expression of proteins associated with cell proliferation and apoptosis. At $24 \mathrm{~h}$ after transfection, western blot analysis was applied to detect the expression levels of the Bcl-2, Bax, Erk, p-Erk, caspase 3 and p53 proteins. YAP, Yes-associated protein; Bcl-2, B-cell lymphoma 2; Bax, Bcl-2-associated X protein; Erk, extracellular signal-regulated kinase; p-, phosphorylated.

that YAP inhibition by YAP siRNA induced Eca-109 cell apoptosis; however, no significant alterations were identified 
in the cell cycle distribution in Eca-109 cells in different groups.

To further examine the mechanisms underlying the effects of YAP inhibition on ESCC cells, the levels of proteins associated with cell proliferation and apoptosis were analyzed. The Bcl-2 family, which is a dominant regulator of programmed cell death in mammalian cells (25), consists of the apoptosis regulator $\mathrm{Bcl}-2$ and its homologues. These proteins govern the mitochondrial outer membrane permeabilization and can be pro-apoptotic (such as Bax, Bcl-2-associated death promoter, $\mathrm{Bcl}-2$ antagonist/killer and Bcl-2 related ovarian killer) or anti-apoptotic (including Bcl-2, Bcl-extra large and Bcl-2-like protein 2). Caspase 3 is the main terminal shear enzyme in the process of cell apoptosis and its activation is prevented by Bcl-2. In the present study, it was observed that the protein expression level of Bcl-2 was significantly decreased in Eca-109 cells transfected with YAP siRNA, while the levels of Bax and caspase 3 were increased. Erk1/2, a member of the mitogen-activated protein kinase family, serves critical roles in regulating cell differentiation, proliferation, migration and angiogenesis through the phosphorylation of phosphatases, cytoskeletal protein and transcriptional factors (26). In addition, $\mathrm{p} 53$ serves a crucial role in preventing cancer formation (27). The present study confirmed that YAP inhibition notably enhanced p53 expression, while the level of p-Erk1/2 was markedly decreased. These results indicate that YAP inhibition affect ESCC cell proliferation and apoptosis at least partly via regulating the expression of Bcl-2, Bax, p-Erk, caspase 3 and p53.

In conclusion, the results presented in the current study demonstrate that YAP gene inhibition suppressed proliferation and induced apoptosis in ECA-109 cells. Furthermore, YAP gene silencing decreased $\mathrm{Bcl}-2$ and $\mathrm{p}$-Erk expression, while the expression of Bax, caspase 3 and p53 was increased. These results indicated that the YAP gene may serve as a novel target in ESCC treatment in the future.

\section{References}

1. Kranzfelder M, Büchler P and Friess H: Surgery within multimodal therapy concepts for esophageal squamous cell carcinoma (ESCC): The MRI approach and review of the literature. Adv Med Sci 54: 158-169, 2009.

2. Chen ZL, Zhao XH, Wang JW, Li BZ, Wang Z, Sun J, Tan FW, Ding DP, Xu XH, Zhou F, et al: microRNA-92a promotes lymph node metastasis of human esophageal squamous cell carcinoma via E-cadherin. J Biol Chem 286: 10725-10734, 2011.

3. Jemal A, Bray F, Center MM, Ferlay J, Ward E and Forman D: Global cancer statistics. CA Cancer J Clin 61: 69-90, 2011.

4. Ma S, Bao JYJ, Kwan PS, Chan YP, Tong CM, Fu L, Zhang N, Tong AHY, Qin YR, Tsao SW, et al: Identification of PTK6, via RNA sequencing analysis, as a suppressor of esophageal squamous cell carcinoma. Gastroenterology 143: 675-686.e12, 2012.

5. Chen W, He Y, Zheng R, Zhang S, Zeng H, Zou X and He J: Esophageal cancer incidence and mortality in China, 2009. J Thorac Dis 5: 19-26, 2013.

6. Lagergren J and Lagergren P: Oesophageal cancer. BMJ 341: c6280, 2010

7. Sant M, Allemani C, Santaquilani M, Knijn A, Marchesi F and Capocaccia R; EUROCARE Working Group: EUROCARE-4. Survival of cancer patients diagnosed in 1995-1999. Results and commentary. Eur J Cancer 45: 931-991, 2009.
8. Liu AM, Xu Z and Luk JM: An update on targeting Hippo-YAP signaling in liver cancer. Expert Opin Ther Targets 16: 243-247, 2012.

9. Hong L, Cai Y, Jiang M, Zhou D and Chen L: The Hippo signaling pathway in liver regeneration and tumorigenesis. Acta Biochim Biophys Sin (Shanghai) 47: 46-52, 2015.

10. Overholtzer M, Zhang J, Smolen GA, Muir B, Li W, Sgroi DC, Deng CX, Brugge JS and Haber DA: Transforming properties of YAP, a candidate oncogene on the chromosome 11q22 amplicon. Proc Natl Acad Sci USA 103: 12405-12410, 2006.

11. Wang X, Su L and Ou Q: Yes-associated protein promotes tumor development in luminal epithelial derived breast cancer. Eur J Cancer 48: 1227-1234, 2012.

12. Ge L, Smail M, Meng W, Shyr Y, Ye F, Fa KH, Li X, Zhou HM and Bhowmick NA: Yes-associated protein expression in head and neck squamous cell carcinoma nodal metastasis. PLoS One 6: e27529, 2011.

13. Cui ZL, Han FF, Peng XH, Chen X, Luan CY, Han RC, Xu WG and Guo XJ: Yes-associated protein 1 promotes adenocarcinoma growth and metastasis through activation of the receptor tyrosine kinase Axl. Int J Immunopathol Pharmacol 25: 989-1001, 2012.

14. Avruch J, Zhou D and Bardeesy N: YAP oncogene overexpression supercharges colon cancer proliferation. Cell Cycle 11: 1090-1096, 2012

15. Wang J, Ma L, Weng W, Qiao Y, Zhang Y, He J, Wang H, Xiao W, Li L, Chu Q, et al: Mutual interaction between YAP and CREB promotes tumorigenesis in liver cancer. Hepatology 58: 1011-1020, 2013.

16. Muramatsu T, Imoto I, Matsui T, Kozaki K, Haruki S, Sudol M, Shimada Y, Tsuda H, Kawano T and Inazawa J: YAP is a candidate oncogene for esophageal squamous cell carcinoma. Carcinogenesis 32: 389-398, 2011.

17. Livak KJ and Schmittgen TD: Analysis of relative gene expression data using real-time quantitative PCR and the 2(-Delta Delta C(T)) method. Methods 25: 402-408, 2001.

18. Liu AM, Wong KF, Jiang X, Qiao Y and Luk JM: Regulators of mammalian Hippo pathway in cancer. Biochim Biophys Acta 1826: 357-364, 2012 .

19. Zender L, Spector MS, Xue W, Flemming P, Cordon-Cardo C, Silke J,Fan ST, Luk JM, Wigler M, Hannon GJ, et al: Identification and validation of oncogenes in liver cancer using an integrative oncogenomic approach. Cell 125: 1253-1267, 2006.

20. Steinhardt AA, Gayyed MF, Klein AP, Dong J, Maitra A, Pan D, Montgomery EA and Anders RA: Expression of Yes-associated protein in common solid tumors. Hum Pathol 39: 1582-1589, 2008.

21. Xu MZ, Yao TJ, Lee NP, Ng IO, Chan YT, Zender L, Lowe SW, Poon RT and Luk JM: Yes-associated protein is an independent prognostic marker in hepatocellular carcinoma. Cancer 115: 4576-4585, 2009

22. Wang Y, Dong Q, Zhang Q, Li Z, Wang E and Qiu X: Overexpression of yes-associated protein contributes to progression and poor prognosis of non-small-cell lung cancer. Cancer Sci 101: 1279-1285, 2010.

23. Dubreuil G, Magliano M, Dubrana MP, Lozano J, Lecomte P, Favery B, Abad P and Rosso MN: Tobacco rattle virus mediates gene silencing in a plant parasitic root-knot nematode. J Exp Bot 60: 4041-4050, 2009.

24. Li JC, Yang XR, Sun HX, Xu Y, Zhou J, Qiu SJ, Ke AW, Cui YH, Wang ZJ, Wang WM, et al: Up-regulation of Krüppel-like factor 8 promotes tumor invasion and indicates poor prognosis for hepatocellular carcinoma. Gastroenterology 139: 2146-2157, 2010.

25. Tsujimoto Y and Shimizu S: Bcl-2 family: Life-or-death switch. FEBS Lett 466: 6-10, 2000.

26. Dhillon AS, Hagan S, Rath O and Kolch W: MAP kinase signalling pathways in cancer. Oncogene 26: 3279-3290, 2007.

27. Surget S, Khoury MP and Bourdon JC: Uncovering the role of p53 splice variants in human malignancy: A clinical perspective. Onco Targets Ther 7: 57-68, 2013. 